\title{
Research on the Constitutive Model for Granular Material
}

\author{
Wei Zhen-hai ${ }^{1,2}$, Wang Meng-shu ${ }^{1} \&$ Zhang Ding-lii \\ ${ }^{1}$ School of Civil Engineering, Beijing Jiaotong University, Beijing, China \\ ${ }^{2}$ School of Rear Professional Service, Logistics Command Academy, Beijing, China \\ Correspondence: Wei Zhen-hai, School of Civil Engineering, Beijing Jiaotong University, Beijing 100044, \\ China.E-mail: wzhai@vip.sina.com
}

Received: June 29, 2012 Accepted: July 13, 2012 Online Published: July 30, 2012

doi:10.5539/esr.v1n2p303 URL: http://dx.doi.org/10.5539/esr.v1n2p303

\begin{abstract}
The constitutive relation of granular aggregate is one important aspect in solid mechanics research, since many problems involved are nothing like the ordinary mechanical problems. Using the energy method, this paper sets up the constitutive model for the granular material through the homogenization of granular material with structural characteristics. Several important factors that are influential to the characteristics of such material are fully considered by this model: performance of granular connector, its geometric position, directional distribution, morphology and size of statistical domain and the density of the connectors. The analysis of the model reveals that the mechanical properties of the granular material are not only related to the properties of the granular material but also to the arrangement of the granular aggregate, i.e. its fabric characteristics. In addition, the morphology of granules determines how the granules will be connected, which in turn modifies the mechanical performance of connection among granules and hence the performance of the granular aggregate. Besides, the couple stress is an important stress component, which makes classical theory no longer applicable to the granular material. Therefore, the constitutive model for the granular material is a generic model reflecting the granular material performance.
\end{abstract}

Keywords: granular material, structural characteristics, homogenization, constitutive model

\section{Introduction}

There is an agreement reached in recent years over the fact that the structural characteristics of granular materials are important factors affecting the macroscopic properties of the materials (Hu, 1999; He \& Shen, 2003; Shi, 1996). Large amount of researches have dealt with the structural properties of soil composed of granules (Hu, 1999; He \& Shen, 2003; Hu, 2000). The microstructure of the material will influence its macroscopic properties, which has also been generally accepted by many. The major problem is how to relate the microscopic properties of the material with its macroscopic properties and to decide what specific microscopic properties actually affect the macroscopic properties, especially the macroscopic mechanical properties. The theoretical study on these problems is still far from adequate, going blindly especially for the microstructure of granular materials. Despite much work done on microscopic parameters of the granular materials, the influence of these parameters on the macroscopic properties remains to be clarified (Zhang, 2008; Shi, 1996). What is important is to set up both micro- and macroscopic models for granular materials to be used in understanding the specific role that each parameter plays. Then we will be able to finally establish the methods for analysis and determination of these microscopic parameters. The paper attempts to establish the homogenization theory for granular structures by using the energy method based on the composition characteristics of the granular material. Next the constitutive model for granular structure on the macroscopic scale under static state is set up in the presence of external force. The static state here refers to that the granular system remains constant in terms of structure under the action of external force. That is to say, there is no slipping or separation (reconnection) among granules in the system, or the system is under elastic state (the dynamic problem is discussed in another paper).

\section{Basic Assumptions}

The performance of the granular system is mainly subject to the influence of connection and composition among the granules. Therefore, the connection among granules is a key factor affecting the macroscopic properties of granular material. In light of this, our discussion is based on the following assumptions:

1) When a stress is applied, the granules themselves will not be damaged, and their surfaces may deform only 
to a negligible amount. The surface layer of the granules can be regarded as elastic, and the interaction among granules is mainly controlled by the performance of this layer;

2) When two granules act upon each other, there is only one point of action (plane or line) (Figure 1);

3) The contact between two granules can be simplified as a connector resembling an elastic beam, which may deform under external force (Figure 2).

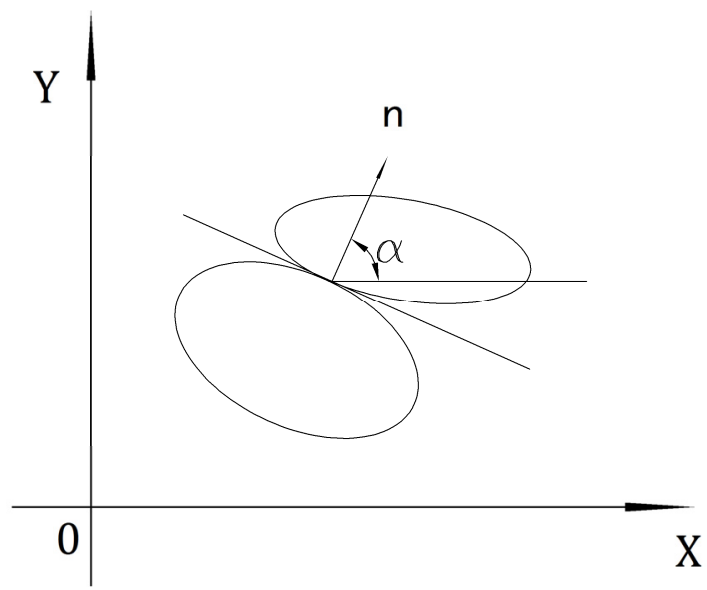

Figure 1. Connection among granules



Figure 2. Simplified model of the connection

According to Assumption 3, the relation of the deformation of the two ends of the connection point with the external force can be written as:

$$
[F]=[K][\delta]
$$

Or in a component form:

$$
\left[\begin{array}{c}
F_{i}^{1} \\
Q_{i}^{1} \\
M_{i}^{1} \\
F_{i}^{2} \\
Q_{i}^{2} \\
M_{i}^{2}
\end{array}\right]=\left[K_{i}^{6 \times 6}\right]\left[\begin{array}{c}
u_{i}^{1} \\
v_{i}^{1} \\
\theta_{i}^{1} \\
u_{i}^{2} \\
v_{i}^{2} \\
\theta_{i}^{2}
\end{array}\right]
$$

This corresponds to the case where the stress acts on the $i^{\text {th }}$ connector, causing deformation, and can be transformed to a global coordinate system:

$$
\left[F_{i}\right]=\left[K_{i}\right]\left[\delta_{i}\right]
$$

where

$$
\begin{gathered}
{\left[F_{i}\right]=\left[F_{i}^{x 1}, F_{i}^{y 1}, M_{i}^{z 1}, F_{i}^{x 2}, F_{i}^{y 2}, M_{i}^{z 2}\right],\left[\delta_{i}\right]=\left[u_{i}^{x 1}, u_{i}^{y 1}, \theta_{i}^{z 1}, u_{i}^{x 2}, u_{i}^{y 2}, \theta_{i}^{z 2}\right]} \\
{\left[K_{i}\right]=\left[R_{i}^{T}\right]^{T}\left[K_{i}^{e}\right]\left[R_{i}^{T}\right]}
\end{gathered}
$$

$\left[R_{i}^{T}\right]$ is the coordinate transformation matrix for the $i^{t h}$ connector. 


\section{Equivalent Energy Homogenization in the Statistical Domain}

The constitutive relation of granular system is subjected to the influence of granular morphology, granular connection and the fabric of granular system. It is a very complex static elastic problem, for which a solution is difficult to find. In order to simplify the problem, we can only estimate the constitutive relation on the macroscopic scale through homogenization. The essence of homogenization is taking an average, i.e. the mean values of parameters on mechanical properties such as stress and strain. The constitutive relation obtained on the macroscopic scale is an equivalent relation represented by the mean values.

Granular material is a highly heterogeneous material because it is composed of granules of variable sizes according to the arrangement in different sections of the material. The connection varies with the position, leading to discrepencies in granular size, gradation and also its performance. In order to get the characteristic parameters of a specific point on the macroscopic scale, the statistical values of the characteristic parameters including granular morphology and connection within a certain area near this point are taken as the representative values; and the area from which the values are taken is know as the statistical domain (Figure 3) (similar to representative unit RAV).

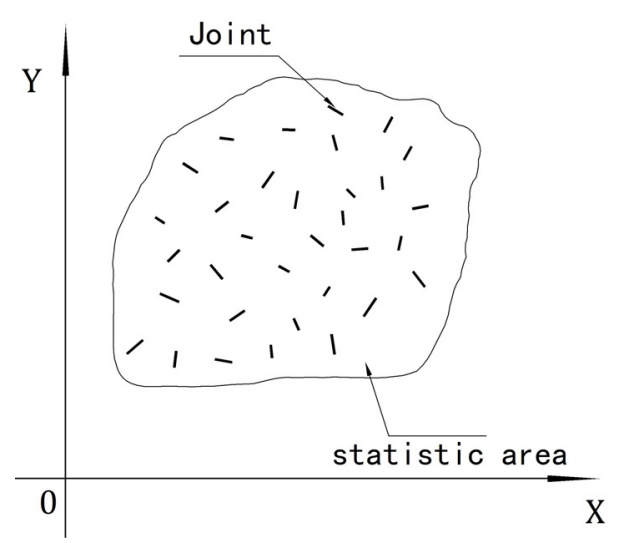

Figure 3. Connection distribution in statistic area

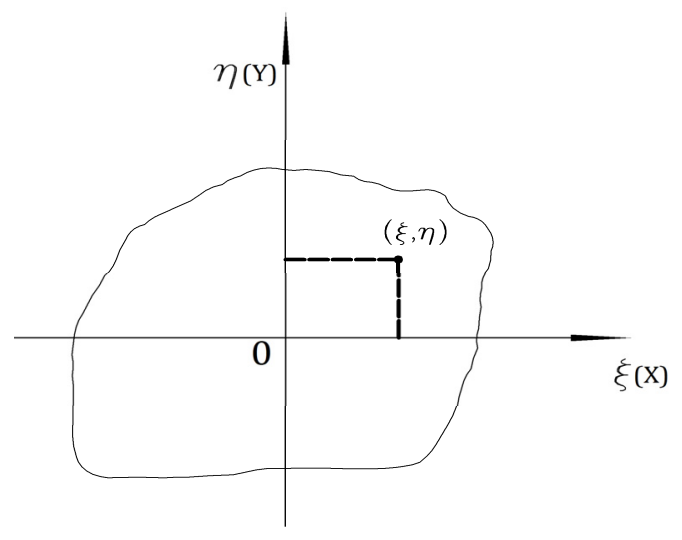

Figure 4. Local coordinates of the connection

There may be many methods to get the representative values of the statistical domain (Hassani \& Hinton, 1998; Xie \& Liao, 2001). But for any of these methods, the equivalent effect principle should be satisfied. Here according to the equivalent energy principle, the following equation is established with $\mathrm{V}$ as the volume of the statistical domain:

$$
\left\{\begin{array}{l}
\varepsilon_{i j}=\frac{1}{V} \int_{V} \varepsilon_{i j}^{*} d V^{*}, \\
\sigma_{i j}=\frac{1}{V} \int_{V} \sigma_{i j}^{*} d V^{*} \\
w_{i j}\left(\varepsilon_{i j}\right)=\frac{1}{V} \int_{V} w_{i j}^{*}\left(\varepsilon_{i j}^{*}\right) d V^{*}
\end{array}\right.
$$

And:

$$
w_{i j}=\frac{1}{2} \sigma_{i j} \varepsilon_{i j}
$$

where $\varepsilon_{i j}, \sigma_{i j}$ and $w_{i j}$ are respectively the average strain, average stress and average strain energy density in the statistical domain. And $\varepsilon_{i j}^{*}, \sigma_{i j}^{*}$ and $w_{i j}^{*}$ are respectively the strain, stress and strain energy density for each connection point in the statistical domain.

The strain energy of the $i^{\text {th }}$ connector is: 


$$
w_{i}=\frac{1}{2}\left[\delta_{i}\right]^{T}\left[K_{i}\right]\left[\delta_{i}\right]
$$

If there are $\mathrm{n}$ connectors in the statistical domain, then the strain energy of the entire domain is given by:

$$
W=\sum_{i=1}^{n} \frac{1}{2}\left[\delta_{i}\right]^{T}\left[K_{i}\right]\left[\delta_{i}\right]
$$

According to the principle of energy conservation, the strain energy (or the work done by the external force) generated from the overall deformation in the statistical domain under external force should be equal to the sum of the strain energy produced from all connectors of the statistical domain; or the average energy density calculated between the two should be equal.

\section{Constitutive Model in the Statistical Domain}

For elastic system composed of uniform medium, the following relation applies:

$$
C_{i j k l}=\frac{\partial^{2} w}{\partial \varepsilon_{i j} \partial \varepsilon_{k l}}, \sigma_{i j}=C_{i j k l} \varepsilon_{k l}
$$

where $\mathrm{w}$ is the strain energy density.

Based on Formula (7) the strain density of the statistical domain can be obtained, and equivalent strain density is found by considering the size of the statiscal domain :

$$
w=\frac{W}{V}=\frac{1}{2 V} \sum_{i=1}^{n}\left[\delta_{i}\right]^{T}\left[K_{i}\right]\left[\delta_{i}\right]
$$

Thus, the above formula can be used to get $C_{i j k l}$, the parameter in equivalent stress-strain relation.

For 2D situation:

$$
[\varepsilon]=\left[\begin{array}{c}
\varepsilon_{11} \\
\varepsilon_{22} \\
\varepsilon_{12} \\
\varepsilon_{21} \\
\varepsilon_{13} \\
\varepsilon_{23}
\end{array}\right]=\left[\begin{array}{c}
u_{1,1} \\
u_{2,2} \\
u_{2,1}-\theta \\
u_{1,2}+\theta \\
\theta_{, 1} \\
\theta_{, 2}
\end{array}\right], \text { and }:[\sigma]=\left[\begin{array}{c}
\sigma_{11} \\
\sigma_{22} \\
\sigma_{12} \\
\sigma_{21} \\
m_{13} \\
m_{23}
\end{array}\right]
$$

The stress-strain relation is represented by:

$$
[\sigma]=[D][\varepsilon]
$$

The strain energy density is known by:

$$
w=w\left(\varepsilon_{11}, \varepsilon_{22}, \varepsilon_{12}, \varepsilon_{21}, \varepsilon_{13}, \varepsilon_{31}\right)=w\left(u_{1,1}, u_{2,2},\left(u_{2,1}-\theta\right),\left(u_{1,2}+\theta\right), \theta_{, 1}, \theta_{, 2}\right)
$$

Thus:

$$
\left\{\begin{array}{l}
\sigma_{11}=\frac{\partial w}{\partial \varepsilon_{11}}=\frac{\partial w}{\partial u_{1,1}}, \sigma_{22}=\frac{\partial w}{\partial \varepsilon_{22}}=\frac{\partial w}{\partial u_{2,2}} \\
\sigma_{12}=\frac{\partial w}{\partial \varepsilon_{12}}=\frac{\partial w}{\partial\left(u_{2,1}-\theta\right)}, \sigma_{21}=\frac{\partial w}{\partial \varepsilon_{21}}=\frac{\partial w}{\partial\left(u_{1,2}+\theta\right)} \\
m_{13}=\frac{\partial w}{\partial \varepsilon_{13}}=\frac{\partial w}{\partial \theta_{, 1}}, m_{23}=\frac{\partial w}{\partial \varepsilon_{23}}=\frac{\partial w}{\partial \theta_{, 2}}
\end{array}\right.
$$

That is to say, if the displacement of the two ends of the connections among all granules in the statistical domain is known, the corresponding deformation energy and deformation energy density can be calculated to obtain the equivalent stress-strain relation, i.e. the equivalent constitutive relation. 
If the statistical domain is a relatively small domain in relation to the granular system, then the displacement of the central point of the statistical domain can be used to approximately represent the displacement of each point of the statistical domain by Taylor expansion:

$$
\left\{\begin{array}{l}
u^{i} \approx u_{0}+L_{\alpha} \frac{\partial u}{\partial t_{\alpha}}+\frac{L_{\alpha}^{2}}{2} \frac{\partial^{2} u}{\partial^{2} t_{\alpha}} \\
\theta^{i} \approx \theta_{0}+L_{\alpha} \frac{\partial \theta}{\partial t_{\alpha}}+\frac{L_{\alpha}^{2}}{2} \frac{\partial^{2} \theta}{\partial^{2} t_{\alpha}}
\end{array}\right.
$$

where $u^{i}$ and $\theta^{i}$ are respectively the displacement of any point of the statiscal domain; $u_{0}$ and $\theta_{0}$ are the macroscopic displacements of the central point of the statistical domain; $L_{\alpha}$ is the distance from the center of the statistical domain; $t_{\alpha}$ is directional derivative.

The expression above is in vector form. Its component form is written as:

$$
\frac{\partial u}{\partial t_{\alpha}}=\frac{\partial}{\partial t_{\alpha}}\left(u_{x} \vec{i}+u_{y} \vec{j}\right)=\left(\frac{\partial u_{x}}{\partial x} \cos \alpha+\frac{\partial u_{x}}{\partial y} \cos \beta\right) \vec{i}+\left(\frac{\partial u_{y}}{\partial x} \cos \alpha+\frac{\partial u_{y}}{\partial y} \cos \beta\right) \vec{j}
$$



where $u_{x}, u_{y}$ and $\theta_{z}$ are respectively the displacement and angular displacement of the central point of the statistical domain in $X, Y$ and $Z$ direction:

When there is a big size difference between the statistical domain and the granular system with neglibile higher order terms, the displacement of the $i^{\text {th }}$ connector in the statistical domain is given by:

$$
\left[\delta_{i}\right]=\left[\begin{array}{c}
u_{x 1}^{i} \\
u_{y 1}^{i} \\
\theta_{z 1}^{i} \\
u_{x 2}^{i} \\
u_{y 2}^{i} \\
\theta_{z 2}^{i}
\end{array}\right]=\left[\begin{array}{l}
u_{x}+\xi_{1}^{i} \frac{\partial u_{x}}{\partial \xi}+\eta_{1}^{i} \frac{\partial u_{x}}{\partial \eta} \\
u_{y}+\eta_{1}^{i} \frac{\partial u_{y}}{\partial \eta}+\xi_{1}^{i} \frac{\partial u_{y}}{\partial \xi} \\
\theta_{z}+\eta_{1}^{i} \frac{\partial \theta_{z}}{\partial \eta}+\xi_{1}^{i} \frac{\partial \theta_{z}}{\partial \xi} \\
u_{x}+\xi_{2}^{i} \frac{\partial u_{x}}{\partial \xi}+\eta_{2}^{i} \frac{\partial u_{x}}{\partial \eta} \\
u_{y}+\eta_{2}^{i} \frac{\partial u_{y}}{\partial \eta}+\xi_{2}^{i} \frac{\partial u_{y}}{\partial \xi} \\
\theta_{z}+\eta_{2}^{i} \frac{\partial \theta_{z}}{\partial \eta}+\xi_{2}^{i} \frac{\partial \theta_{z}}{\partial \xi}
\end{array}\right]
$$

After sorting out: 


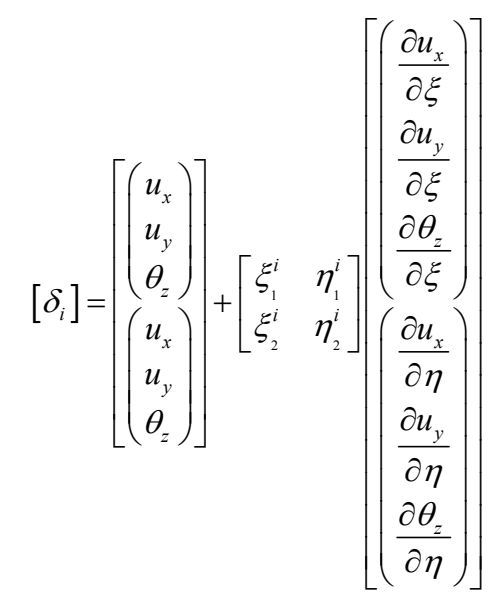

$$
\begin{aligned}
& \text { If } \mathbf{u}_{0}^{\mathbf{T}}=\left[\begin{array}{lll}
u_{x} & u_{y} & \theta_{z}
\end{array}\right]^{T}, \text { then: } \begin{aligned}
\frac{\partial \mathbf{u}_{0}^{\mathbf{T}}}{\partial \xi} & =\left[\begin{array}{lll}
\frac{\partial u_{x}}{\partial \xi} & \frac{\partial u_{y}}{\partial \xi} & \frac{\partial \theta_{z}}{\partial \xi}
\end{array}\right]^{T} \\
\frac{\partial \mathbf{u}_{0}^{\mathbf{T}}}{\partial \eta} & =\left[\begin{array}{lll}
\frac{\partial u_{x}}{\partial \eta} & \frac{\partial u_{y}}{\partial \eta} & \frac{\partial \theta_{z}}{\partial \eta}
\end{array}\right]^{T}
\end{aligned}
\end{aligned}
$$

Formula (16) can be simplified as:

$$
\left[\delta_{i}\right]=\left[\begin{array}{l}
\mathbf{u}_{\mathbf{0}} \\
\mathbf{u}_{\mathbf{0}}
\end{array}\right]+\left[\begin{array}{ll}
\xi_{1}^{i} & \eta_{1}^{i} \\
\xi_{2}^{i} & \eta_{2}^{i}
\end{array}\right]\left[\begin{array}{c}
\frac{\partial \mathbf{u}_{\mathbf{0}}}{\partial \xi} \\
\frac{\partial \mathbf{u}_{\mathbf{0}}}{\partial \eta}
\end{array}\right]
$$

Substitution into the expression of strain energy yields:

$$
\begin{aligned}
w_{i}= & \frac{1}{2}\left[\delta_{i}\right]^{T}\left[K_{i}\right]\left[\delta_{i}\right] \\
& =\frac{1}{2}\left\{\mathbf{u}_{\mathbf{0}}\left[\begin{array}{l}
1 \\
1
\end{array}\right]+\left[\begin{array}{ll}
\xi_{1}^{i} & \eta_{1}^{i} \\
\xi_{2}^{i} & \eta_{2}^{i}
\end{array}\right]\left[\begin{array}{c}
\frac{\partial \mathbf{u}_{\mathbf{0}}}{\partial \xi} \\
\frac{\partial \mathbf{u}_{\mathbf{0}}}{\partial \eta}
\end{array}\right]\right\} \\
& {\left[K_{i}\right]\left\{\mathbf{u}_{\mathbf{0}}\left[\begin{array}{l}
1 \\
1
\end{array}\right]+\left[\begin{array}{ll}
\xi_{1}^{i} & \eta_{1}^{i} \\
\xi_{2}^{i} & \eta_{2}^{i}
\end{array}\right]\left[\begin{array}{c}
\frac{\partial \mathbf{u}_{\mathbf{0}}}{\partial \xi} \\
\frac{\partial \mathbf{u}_{\mathbf{0}}}{\partial \eta}
\end{array}\right]\right\} }
\end{aligned}
$$

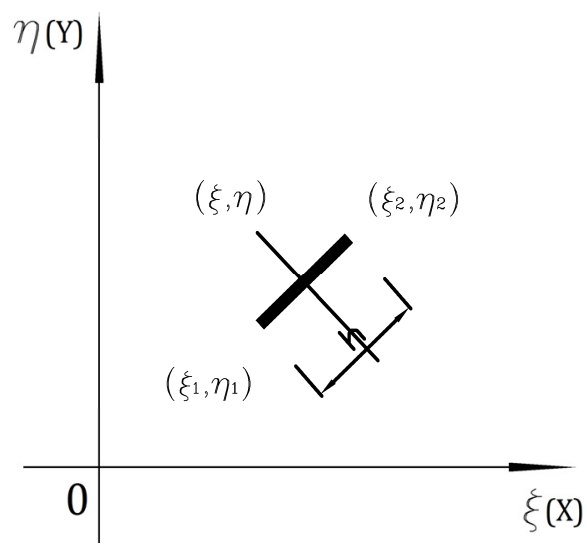

Figure 5. Connection parameters 
In consideration that the size of the $2 \mathrm{D}$ statistical domain is $\mathrm{A}_{\mathrm{c}}$, the summation of strain energy of all connectors in statistical domain is given by:

$$
w=\frac{1}{2 A_{c}}\left\{\mathbf{u}_{\mathbf{0}}^{\mathrm{T}} \overline{\mathbf{K}}_{\mathbf{1}} \mathbf{u}_{\mathbf{0}}+\mathbf{u}_{\mathbf{0}}^{\mathrm{T}} \overline{\mathbf{K}}_{2} \boldsymbol{\varepsilon}_{\mathbf{0}}+\boldsymbol{\varepsilon}_{\mathbf{0}}^{\mathrm{T}} \overline{\mathbf{K}}_{3} \mathbf{u}_{\mathbf{0}}+\boldsymbol{\varepsilon}_{\mathbf{0}}^{\mathrm{T}} \overline{\mathbf{K}}_{4} \boldsymbol{\varepsilon}_{0}\right\}
$$

where $\mathbf{u}_{\mathbf{0}}$ is the same as $\boldsymbol{\varepsilon}_{\mathbf{0}}=\left[\begin{array}{ll}\frac{\partial \mathbf{u}_{0}}{\partial \xi} & \frac{\partial \mathbf{u}_{0}}{\partial \eta}\end{array}\right]^{T}$

$$
\left\{\begin{array}{l}
\overline{\mathbf{K}}_{\mathbf{1}}=\left[\begin{array}{ll}
1 & 1
\end{array}\right] \sum_{i=1}^{n} \mathbf{K}_{\mathbf{i}}\left[\begin{array}{l}
1 \\
1
\end{array}\right] \\
\overline{\mathbf{K}}_{\mathbf{2}}=\sum_{i=1}^{n}\left[\begin{array}{ll}
1 & 1
\end{array}\right] \mathbf{K}_{\mathbf{i}}\left[\begin{array}{ll}
\xi_{1}^{i} & \eta_{1}^{i} \\
\xi_{2}^{i} & \eta_{2}^{i}
\end{array}\right] \\
\overline{\mathbf{K}}_{\mathbf{3}}=\sum_{i=1}^{n}\left[\begin{array}{ll}
\xi_{1}^{i} & \eta_{1}^{i} \\
\xi_{2}^{i} & \eta_{2}^{i}
\end{array}\right] \mathbf{K}_{\mathbf{i}}\left[\begin{array}{ll}
1 & 1
\end{array}\right] \\
\overline{\mathbf{K}}_{\mathbf{4}}=\sum_{i=1}^{n}\left[\begin{array}{ll}
\xi_{1}^{i} & \eta_{1}^{i} \\
\xi_{2}^{i} & \eta_{2}^{i}
\end{array}\right] \mathbf{K}_{\mathbf{i}}\left[\begin{array}{ll}
\xi_{1}^{i} & \eta_{1}^{i} \\
\xi_{2}^{i} & \eta_{2}^{i}
\end{array}\right]
\end{array}\right.
$$

$\mathbf{K}_{\mathbf{i}}$ is the stiffness matrix after $3 \times 3$ segmentation:

$$
\begin{aligned}
\mathbf{K}_{\mathbf{i}} & =\left[\begin{array}{ll}
K_{11}^{i} & K_{12}^{i} \\
K_{21}^{i} & K_{22}^{i}
\end{array}\right], K_{11}^{i}=\left[\begin{array}{lll}
k_{11} & k_{12} & k_{13} \\
k_{21} & k_{22} & k_{23} \\
k_{31} & k_{32} & k_{33}
\end{array}\right] \\
K_{12}^{i} & =\left[\begin{array}{lll}
k_{14} & k_{15} & k_{16} \\
k_{24} & k_{25} & k_{26} \\
k_{34} & k_{35} & k_{36}
\end{array}\right], K_{21}^{i}=\left[\begin{array}{lll}
k_{41} & k_{42} & k_{43} \\
k_{51} & k_{52} & k_{53} \\
k_{61} & k_{62} & k_{63}
\end{array}\right], \\
K_{22}^{i} & =\left[\begin{array}{lll}
k_{44} & k_{45} & k_{46} \\
k_{54} & k_{55} & k_{56} \\
k_{64} & k_{65} & k_{66}
\end{array}\right]
\end{aligned}
$$

From Formula (18) we can see that the strain energy density is related to the displacement and strain as well as to connector stiffness $\mathrm{K}_{\mathrm{i}}$, which depends on the morphology of granules and the action force among granules. $\overline{\mathbf{K}}_{1}, \overline{\mathbf{K}}_{2}, \overline{\mathbf{K}}_{3}, \overline{\mathbf{K}}_{4}$ represent not only the action among granular connectors but also the influence of the position on the granular connector. The constitutive relation of the granules varies according to the specific position.

The equivalent constitutive relation in the statistical domain can be derived based on strain energy density from Formula (12):

$$
\left[\begin{array}{l}
\sigma_{11} \\
\sigma_{21} \\
m_{13} \\
\sigma_{21} \\
\sigma_{22} \\
m_{23}
\end{array}\right]=\frac{1}{A_{c}}\left\{\overline{\mathbf{K}}_{\mathbf{3}} \mathbf{u}_{\mathbf{0}}+\overline{\mathbf{K}}_{\mathbf{4}} \boldsymbol{\varepsilon}_{\mathbf{0}}\right\}
$$

The derivative of the composite function is taken with respect to $\sigma_{12}$ and $\sigma_{21}$.

From formula (19) it can be seen that the stress and couple stress are not only related to the strain but also to the displacement. We can prove that when stiffness matrix $\mathrm{K}_{\mathrm{i}}$ among granules can be simplified as a unit matrix of beam, the couple stress is independent of angle $\theta_{\mathrm{z}}$ and other stress components are also not related to the displacement. However, if coordinate transformation is required for the unit matrix of beam, the expression of each stress component will include the displacement components.

Formula (19) shows that the equivalent constitutive relation in the statistical domain is determined by $\overline{\mathbf{K}}_{3}$ and $\overline{\mathbf{K}}_{4}$, which are in turn related to two factors: one is $\mathrm{K}_{\mathrm{i}}$, the stiffness matrix of granular connector, affected by the interaction among granules; another is the position of the connector in the statistical domain, affected by the position and direction of the connector in the statistical domain. Apparently, the constitutive relation of the 
granular material is subjected to the influence of both the connection properties of granules and the distribution of the connectors among granules microscopically.

For the convenience of calculation, the coordinates of the midpoint of the connector is assumed as $(\xi, \eta)$, its length $h_{i}$; and the angles with respect to $X$ and $Y$ axis are $\alpha$ and $\beta$ respectively. Thus:

$$
\left\{\begin{array} { l } 
{ \xi _ { 1 } ^ { i } = \xi _ { i } + \frac { h _ { i } } { 2 } \operatorname { c o s } \alpha _ { i } } \\
{ \xi _ { 2 } ^ { i } = \xi _ { i } - \frac { h _ { i } } { 2 } \operatorname { c o s } \alpha _ { i } }
\end{array} \text { and } \left\{\begin{array}{l}
\eta_{1}^{i}=\eta_{i}+\frac{h_{i}}{2} \cos \beta_{i} \\
\eta_{2}^{i}=\eta_{i}-\frac{h_{i}}{2} \cos \beta_{i}
\end{array}\right.\right.
$$

If

$$
\mathbf{G}_{\mathbf{i}}=\left[\begin{array}{cc}
\xi_{i}+\frac{h_{i}}{2} \cos \alpha_{i} & \xi_{i}-\frac{h_{i}}{2} \cos \alpha_{i} \\
\eta_{i}+\frac{h_{i}}{2} \cos \beta_{i} & \eta_{i}-\frac{h_{i}}{2} \cos \beta_{i}
\end{array}\right]
$$

Then:

Here $\mathrm{I}=\left[\begin{array}{ll}1 & 1\end{array}\right]^{\mathrm{T}}$.

$$
\left\{\begin{array}{l}
\overline{\mathbf{K}}_{\mathbf{3}}=\sum_{i=1}^{n} \mathbf{G}_{\mathbf{i}} \mathbf{K}_{\mathbf{i}} \mathbf{I} \\
\overline{\mathbf{K}}_{4}=\sum_{i=1}^{n} \mathbf{G}_{\mathbf{i}} \mathbf{K}_{\mathbf{i}} \mathbf{G}_{\mathbf{i}}^{\mathbf{T}}
\end{array}\right.
$$

Obviously, $\mathbf{G}_{\mathbf{i}}$ is the spatial characteristic matrix for the connector that includes three parameters: position, angle and length of the connector. Thus, statistical process is necessary for the three parameters when we analyze the distribution characteristics of the granular material.

\section{Discussion}

Formula (19) gives the equivalent constitutive relation of the granular material. In the following, the meaning of the parameters in this relation and their respective roles will be examined.

\subsection{Meaning of the Parameters}

Formula (20) can be decomposed into:

$$
\begin{aligned}
\mathbf{G}_{\mathbf{i}} & =\left[\begin{array}{ll}
\xi_{i} & \xi_{i} \\
\eta_{i} & \eta_{i}
\end{array}\right]+\frac{h_{i}}{2}\left[\begin{array}{ll}
\cos \alpha_{i} & -\cos \alpha_{i} \\
\cos \beta_{i} & -\cos \beta_{i}
\end{array}\right] \\
& =\left[\begin{array}{l}
\xi_{i} \\
\eta_{i}
\end{array}\right]\left[\begin{array}{ll}
1 & 1
\end{array}\right]+\frac{h_{i}}{2}\left[\begin{array}{ll}
\cos \alpha_{i} \\
\cos \beta_{i}
\end{array}\right]\left[\begin{array}{ll}
1 & -1
\end{array}\right]
\end{aligned}
$$

By substituting into Formula (21), $\overline{\mathbf{K}}_{3}=\sum_{i=1}^{n}\left\{\left[\begin{array}{l}\xi_{i} \\ \eta_{i}\end{array}\right] \otimes \mathbf{K}_{1}^{i}+\frac{h_{i}}{2}\left[\begin{array}{c}\cos \alpha_{i} \\ \cos \beta_{i}\end{array}\right] \otimes \mathbf{K}_{2}^{i}\right\}$

$$
\overline{\mathbf{K}}_{4}=\sum_{i=1}^{n}\left\{\begin{array}{l}
{\left[\begin{array}{c}
\xi_{i} \\
\eta_{i}
\end{array}\right]\left[\begin{array}{ll}
\xi_{i} & \eta_{i}
\end{array}\right] \otimes \mathbf{K}_{1}^{i}+\frac{h_{i}}{2}\left[\begin{array}{l}
\cos \alpha_{i} \\
\cos \beta_{i}
\end{array}\right]\left[\begin{array}{ll}
\xi_{i} & \eta_{i}
\end{array}\right] \otimes \mathbf{K}_{2}^{i}} \\
+\frac{h_{i}}{2}\left[\begin{array}{l}
\xi_{i} \\
\eta_{i}
\end{array}\right]\left[\begin{array}{ll}
\cos \alpha_{i} & \cos \beta_{i}
\end{array}\right] \otimes \mathbf{K}_{3}^{i} \\
+\left(\frac{h_{i}}{2}\right)^{2}\left[\begin{array}{l}
\cos \alpha_{i} \\
\cos \beta_{i}
\end{array}\right]\left[\begin{array}{ll}
\cos \alpha_{i} & \cos \beta_{i}
\end{array}\right] \otimes \mathbf{K}_{4}^{i}
\end{array}\right\}
$$

Where

$$
\mathbf{K}_{1}^{i}=K_{11}^{i}+K_{12}^{i}+K_{21}^{i}+K_{22}^{i} ; \quad \mathbf{K}_{2}^{i}=K_{11}^{i}+K_{12}^{i}-K_{21}^{i}-K_{22}^{i} ;
$$




$$
\mathbf{K}_{3}^{i}=K_{11}^{i}-K_{12}^{i}+K_{21}^{i}-K_{22}^{i} \text { and } \mathbf{K}_{4}^{i}=K_{11}^{i}-K_{12}^{i}-K_{21}^{i}+K_{22}^{i}
$$

Where $K_{11}^{i} 、 K_{12}^{i} 、 K_{21}^{i} 、 K_{22}^{i}$ are the block submatrices of the stiffness matrix of the connector respectively.

If the stiffness matrix of the connector is assumed to be similar to that of the elastic beam, then

$$
K^{6 \times 6}=\left[\begin{array}{ll}
K_{11}^{3 \times 3} & K_{12}^{3 \times 3} \\
K_{21}^{3 \times 3} & K_{22}^{3 \times 3}
\end{array}\right]
$$

Where

$$
\begin{aligned}
K_{11} & =\left[\begin{array}{lll}
k_{11} & k_{12} & k_{13} \\
k_{21} & k_{22} & k_{23} \\
k_{31} & k_{32} & k_{33}
\end{array}\right], \\
K_{12} & =K_{21}^{T}=\left[\begin{array}{lll}
-k_{11} & -k_{12} & k_{13} \\
-k_{21} & -k_{22} & k_{23} \\
-k_{31} & -k_{32} & 1 / 2 k_{33}
\end{array}\right], \\
K_{22} & =\left[\begin{array}{ccc}
k_{11} & k_{12} & -k_{13} \\
k_{21} & k_{22} & -k_{23} \\
-k_{31} & -k_{32} & k_{33}
\end{array}\right]
\end{aligned}
$$

Thus,

$$
\begin{aligned}
\mathbf{K}_{1}^{i} & =\left[\begin{array}{ccc}
0 & 0 & 0 \\
0 & 0 & 0 \\
0 & 0 & 3 k_{33}
\end{array}\right], \mathbf{K}_{2}^{i}=\left[\begin{array}{ccc}
0 & 0 & 4 k_{13} \\
0 & 0 & 4 k_{23} \\
0 & 0 & 0
\end{array}\right], \\
\mathbf{K}_{3}^{i} & =\left[\begin{array}{ccc}
0 & 0 & 0 \\
0 & 0 & 0 \\
4 k_{13} & 4 k_{13} & 0
\end{array}\right], \mathbf{K}_{4}^{i}=\left[\begin{array}{ccc}
4 k_{11} & 4 k_{12} & 0 \\
4 k_{21} & 4 k_{22} & 0 \\
0 & 0 & 0
\end{array}\right]
\end{aligned}
$$

By substituting into the expressions of $\overline{\mathbf{K}}_{3}, \overline{\mathbf{K}}_{4}$, it is found that for $\overline{\mathbf{K}}_{3}$ only the third column is non-zero; it is the angular displacement that corresponds to the displacement matrix. That is to say, what $\overline{\mathbf{K}}_{3}$ represents is the stress changes due to rotational displacement, since translational displacement will not cause stress changes. Then we examine the influence of the statistical domain. If the position of the connector with respect to the coordinate origin is symmetrical having the same stiffness matrix for each connector, then $\sum_{i=1}^{n} \xi_{i}=\sum_{i=1}^{n} \eta_{i}=0$, which means rotational displacement will not cause the couple stress. Meanhile, $\sum_{i=1}^{n} \cos \alpha_{i}=\sum_{i=1}^{n} \cos \beta_{i}=0$ will not lead to normal stress and shear stress components. Thus, $\overline{\mathbf{K}}_{3}$ represents the changes of stress components resulting from statistically eccentric and non-uniform action. The classical theory generally assumes that the material is uniform and continuous, and the material can be regarded as completely symmetrical, especially when the stress state of a point is considered. Therefore, the stress problems arising from the displacement of a non-uniform rigid body cannot be accounted for.

$\overline{\mathbf{K}}_{4}$ in the formula is composed of four terms. For the first term, only the subterm that is related to the angle gradient in the influence maxtirx of the connector is non-zero. So the term is the mean value of the couple stress components in the changing statistical domain of each point caused by angle gradient. For the influence matix in the second item, only the third column is non-zero, which is only related to the angle gradient when the arrangement of strain vector is considered. Therefore, the second term is in fact represents the contribution of angle gradient to other stress components. The contribution of strain to couple stress component can be found by examining the influence matix of the third term. For the influence matrix of the last term, only the four positions towards the upper left corner have values, while the rows and columns related to the angle have none. This indicates that the term represents the relation of other strain componments with the corresponding stress 
components except the angle. In addition, if the morphology of the statistical domain and the stiffness matrix of the connector are symmetrical, we will find that the second and the third terms may be zero. This indicates that the two terms are related to the coordinates as well as the asymmetry of the system. This is an important feature of the constitutive relation of granular materials that is not represented in the classical theory.

\subsection{Further Analysis of Influential Parameters}

From the expressions of $\overline{\mathbf{K}}_{3}$ and $\overline{\mathbf{K}}_{4}$ it can be found that the influential parameters are basically related to the following three aspects:

1) The characteristics of granular connectors;

2) The geometric position, direction and distribution of granular connectors;

3) The morphology and size of the statistical domain, as well as the density of the connectors in the statistical domain.

The mechanical interaction among granules is influenced by the characteristics of granular connectors. The higher the stiffness of the connector, the higher the equivalent stiffness in the statistical domain will be. And the stiffness of the connector is also affected by the characteristics of the connection plane. For instance, the plane with friction action is very different from the plane with cementing action. Even the connection planes of the same type have variable stiffness characteristics due to the morphology of the connection plane. The plane with friction action has different impact for point-to-point contact and plane-to-plane contact. The anti-bending performance of the plane-to-plane contact is much higher than that of point-to-point contact.

The geometic position and directional distribution of granular connectors reflect the fabric relation of granules, which is an important aspect that affects the properties of granular materials. The geometic position and directional distribution are two important parameters that quantitatively describe the properties of granular materials. However, we have not yet understood how structural parameters of granular materials affect its macroscopic mechanical properties. Through the analysis of the constitutive relation of granular materials it can be found that the position of the granular connectors and the connection direction are two parameters that reflect the fabric characteristics of granular material.

The morphology and size of the statistical domain are also important influential parameters for the equivalent constitutive relation. The discrepancy in the morphology and size of the statistical domain may lead to different results. And this characteristic related to morphology and size of the statistical domain is also different from what is implied in the classical theory. That is, the characteristics of granular material have certain scale effect. Even given the same morphology and size of the statistical domain, the quantity of the connectors in the statistical domain or the density of the connectors may be greatly different due to different granular scale in the statistical domain. This explains the difference in the calculation results.

\section{Conclusion}

Through the above analysis we can see that the constitutive relation of the granular material is very complex and have much more influential factors compared to uniform and continuous materials. After the analysis of the model, conclusions that are very different from those obtained for uniform and continuous materials based on classical theory are reached:

1) Several important factors influencing the performance of granular material are fully considered in the constitutive model for the granular material: the performance of the connectors, geometic position and directional distribution, morphology and size of the statistical domain and the density of the connectors. The role of these factors can be analyzed quantitatively by this model;

2) The constitutive model for granular material is a generic model that reflects the material performance. The parameters in the model represent the properties of granular materials quite comprehensively. When some parameters degenerate to a homogenization state, the model is automatically adapted to the classical theory;

3) We have found out relevant parameters that can fully reflect the essential properties of granular structures by the model. This lays a theoretical foundation for validation tests of granular materials by reducing the blindness of parameter screening for the tests;

4) The equivalent stress-strain relation of granular material is directly related to the positon and directional distribution of granular connectors. This means that the non-uniform distribution of the connectors will influence the equivalent constitutive relation. If the properties of granular material are studied by assuming that the granules are continuous, then geometric position and directional distribution of the connectors will inevitably affect the constitutive relation; 
5) Model analysis reveals that the couple stress in the equivalent constitutive relation of granular materials is an important stress component, which is not otherwise accounted for in classical theory. Therefore, the analysis method used in classical theory can not be directly borrowed for their applications in macroscopic study of granular materials. Cosserat theory is recommended for such cases since it considers the effect of the couple stress.

This paper has proposed and discussed the constitutive model for granular materials, but the research is still preliminary. Many problems need to be more thoroughly investigated, especially the description of the distribution of the connectors and the influence of granular morphology on the connection properties.

\section{References}

Cao Xiao-Qing, Wang Phi-Hua, Ma Honk-Wei, Zhao Long-Mao, \& Yang Gui-Tong. (2006). The Effects of Micro-Structure on the Equivlent Elastic Constants of Cellular Materials. Journal of Taiyuan University of Technology, 37(1), 1-5.

Chen Huifa. (2004). Elasticity and Plasticity. China Architecture \& Building Press July.

Hassani, B., \& Hinton, E. (1998a). A review of homogenization and topology optimization I-homogenization theory for media with periodic structure. Computers and Structures, 69, 707-717. http://dx.doi.org/10.1016/S0045-7949(98)00131-X

Hassani, B., \& Hinton, E. (1998b). A review of homogenization and topology optimization II-analytical and numerical solution of homogenization equations. Computers and Structures, 69, 719-738. http://dx.doi.org/10.1016/S0045-7949(98)00132-1

Hassani, B., \& Hinton, E. (1998c). A review of homogenization and topology optimization III topology optimization using optimality criteria. Computers and Structures, 69, 739-756. http://dx.doi.org/10.1016/S0045-7949(98)00133-3

He Kai-Sheng, \& Shen Zhu Jian. (2003). Study on Micro-Deformation and Mechanism of Structural Clay. Journal of Hohai University, 31(2), 161-165.

$\mathrm{Hu}$ Ruilin. (1999). Microstructure Effect on the Subsdnce of Loess. Journal of Engineering Geology, 17(2), 161-167.

$\mathrm{Hu}$ Ruilin. (2000). Approach to the Strength Behaviors and Microstructural Changes of Loess under Dynamic Load. Chinese Journal of Geotechnical Engineering, 22(2), 177-181.

$\mathrm{Hu}$ Xin, Sun Qiu, Hong Bao-Ning, \& Zhou Yu-Quan. (2006). Correlations between Compressibility and Micro-structural Form of Cohesive Soils. China Harbour Engineering, 145(5), 16-19.

Jtang Hong-Ying, Lu Jin-Bu, \& Miao Tian-De. (2008). An Experimental Analysis and the Establishment of Theoretical Models of the Granular Structures. Journal of Gansu Sciences, 20(1), 135-138.

Ju Hai-Yan, Huang Hong-Yuan, Li Jian-Hua, \& Yang Wei-Zhong. (2006). The Study on the Structural Constitutive Models of Soil and Rock. China Tungsten Industry, 21(4), 21-24.

Kaneko, K., Terada, K., Kyoya, T., \& Kishino, Y. (2003). Global-Local Analysis of Granular Media in Quasi-Static Equilibrium. International Journal of Solids And Structures, 40, 4043-4069. http://dx.doi.org/10.1016/S0020-7683(03)00209-9

Kang Xin, \& Xi Zhanwen. (2007). Size Effect on the Dynamic Characteristic of a Micro Based on Cosserat Theory. Journal of Mechanical Strength, 29(1), 1-4.

Li Shun-Qun, Zheng Gang, Zhao Rui-Bin, \& Wang Pei. (2009). Correlation Analysis and Principal Component Analysis on Microstructure Parameters of Clay. Chinese Journal of Geotechnical Engineering, 31(7), 1120-1126.

Li Xiang-Quan, Hu Rui-Lin, \& Zhang Li. (2000). The Variation of Microstructure during Soft Soil Solidification. Earth Science Frontiers (China University of Geosciences, Beijing), 7(1), 147-152.

Liu Xu, Wang Lan-Min, Chen Long-Zhu, \& Sun Jun-Jie. (2007). A Volumetric Model of Loess Based On Microstructure Instability and Generalized Suction Theories. Rock and Soil Mechanics, 28(12).

Patrice, C., \& Tanguy, M. (2006). Computational Homogenization of Periodic Beam-Like Structures. International Journal of Solids and Structures, 43, 686-696. http://dx.doi.org/10.1016/j.ijsolstr.2005.03.063

Shi Bin. (1996). Review and Prospect on the Microstructure of Clayey Soil. Journal of Engineering Geology, 4, $39-45$. 
Sun Qiang, Zhang Xiao-Ke, \& Li Hou-En. (2008). Research on Microstructural Catastrophe Model of Deformation of Collapsible Loess. Rock and Soil Mechanics, 29(3), 663-672.

Tang Liansheng, Liao Huarong, \& Zhang Qinghua. (2006). Structural Entropy of Soil and Quantitative Research for Soil Structure Characteristics. Chinese Journal of Rock Mechanics and Engineering, 25(10), 1997-2002.

Wang Jun-Chao, Jia Yong-Gang, Shi Wen-Jun, Shan Hong-Xian, Xu Guo-Hui, \& Fu Yuan-Bin. (2004). Case Study on the Fractal Characteristic Variations of Silty Soil Microstructure Due to Differential Hrodynamics in the Yellow River Estuarine Area. Advances in Marine Science, 22(2), 177-183.

Wang Wei, Feng Xiao-Ping, Chen Yan, \& Wang Jian. (2006). Analysis of Intrinsic Correlation of Mechanical Strength with Microstructure Dynamic Environmental Energy Field of Clayey Soils. Rock and Soil Mechanics, 27(12), 2219-2224.

Wanga, J. G., Leungb, C. F., \& Ichikawa, Y. (2002). A Simplified Homogenization Method for Composite Soils. Computers and Geotechnics, 29, 477-500. http://dx.doi.org/10.1016/S0266-352X(02)00004-6

Wei Lide. (2004). Study on Rock Mechanics Damage and Rheology Constitutive Models. Chinese Journal of Rock Mechanics And Engineering, 23(24), 4265-4265.

Weihong Zhang, Gaoming Dai, Fengwen Wang, Shiping Sun, \& Hicham Bassir. (2007). Using Strain Energy-Based Prediction of Effective Elastic Properties in Topology Optimization of Material Microstructures. Acta Mech Sin, 23, 77-89. http://dx.doi.org/10.1007/s10409-006-0045-2

Xiao Qi-lin, Ling Zhong, Wu Yong-Li, \& Yao Wen-hui. (2003). Incompatible Finite Element Analysis of Couple-stress Problems. Journal of the Graduate School of the Chinese Academy of Sciences, 20(2), 1-9.

Xie Xianhai, \& Liao Daoxun. (2001). Computation Method of Equivalent Elastic Tensor in Homogenization Method.

Yasser, M., \& Shabana, N. N. (2008). Numerical Evaluation of the Thermomechanical Effective Properties of a Functionally Graded Material Using the Homogenization Method. International Journal of Solids And Structures, 45, 3494-3506. http://dx.doi.org/10.1016/j.ijsolstr.2008.02.012

Yuan Zhen, Wu Changchun, \& Li Hua. (2003). Homogenization-Based Topology Design for Pure Torsion of Composite Shafts. Acta Mechanica Sinica, 19(3), 0567-7718.

Zhang Hong Wu. (2001a). Hom Ogenization M Ethod for the Analysis of Assemblage of Elastic Contact Grains Part One: Local Rve Analysis. Acta Materiae Compositae Sinicavol, 4, 94-97.

Zhang Hong Wu. (2001b). Homogenisation Method for the Analysis of Assemblage of Elastic Contactgrains, Part II-Macro Homogenisation Analysis. Acta Materiae Compositae Sinicavol, 4, 99-102.

Zhang Hua Jiel, Sun Qiu, Hu Xin, \& Hong Bao Ning. (2008). Summarizing Study on the Methods of Testing Microstructure of Geomaterials. Soil Eng. and Foundation, 22(4), 50-54.

Zhang Li-Zhong, Hu Rui-Lin, Li Xiang-Quan, \& Zhanu Yong-Bo. (2008). Soil Microstructure Quantitative Analysis System and Its Application. Geological Science and Technology Information, 27, 108-112.

Zhang Min-Jiang, Yan Jing, \& Chu Hong-Xia. (2005). The Quantitative Analysis of Microstructure Parameter of Soft Soil. Journal of Shenyang Jianzhu University (Natural Science), 21(5), 455-459.

Zhang Pei-Yuan, Zhang Xia-Min, \& Tan Guan-Pu. (2002). A Difficulty of Symmetrical Stress Model. Journal of Chongqing University (Natural Science Edition), 25(4), 8-9. 